\title{
The association between HLA alleles (A, B, DRB1) and HIV-1 infection/ AIDS progression in the Han Chinese population of Hubei province
}

\author{
Wangxia Li $\dagger^{*}$, Ru Yang $\dagger$, Fengwu Qiu $\dagger$, Jing Xu, Gang Shen, Yan Ma \\ Blood Center of Wuhan, Wuhan, Hubei 430030, China
}

\begin{abstract}
The association between HLA alleles (A, B, DRB1), haplotypes and AIDS progression in HIV-1 infected patients was investigated by analyzing and comparing the differences gene frequencies of HLA alleles (A, B, DRB1) and haplotypes in HIV-1 infected and AIDS individuals in Hubei province of China. Four hundred and twentyfour HIV-1 seropositive individuals were divided into two groups: HIV-1 infected group and AIDS patient group, according to diagnostic criteria. HLA-A, B, and DRBl allele typing was performed using polymerase chain reaction-sequence-specific oligonucleotide (PCR-SSOP) and polymerase chain reaction-sequencing based typing (PCR-SBT) techniques. Our study revealed that $\mathrm{B} * 57: 01$ seemed resistant to AIDS progression, and the presence of DRB1*04:05 was associated with a poor disease outcome in HIV-1 infection. These associations were independent of age, sex, and transmission route of the host. No association was observed between HLA-A, B, DRB1 homozygotes, HLA-Bw4, Bw6 serological types and AIDS progression. We concluded that HLA gene polymorphism has a significant role in HIV-1 infection/AIDS progression. This observational study may open up avenues for precision medicine in the personalized prevention and treatment of AIDS.
\end{abstract}

Keywords: HLA, HIV-1, defense gene, resistance gene, risk factor, disease progression

\section{INTRODUCTION}

HIV-1 infected individuals show different disease processes. More than 95 percent of people infected with HIV-1 will develop AIDS in 2 to 10 years. On the contrary, the remaining 5 percent will show no characteristic symptoms of AIDS, and usually maintain a normal amount of $\mathrm{CD} 4^{+} \mathrm{T}$ cells without antiviral treatment over 10 years ${ }^{[1]}$. HLA alleles have been reported to be associated with AIDS progression ${ }^{[2-5]}$. However, results were controversial between HLA allele groups due to regional and ethnic diversity ${ }^{[6-9]}$. Subsequently the association between HLA allele polymorphism and AIDS progression still remains elu-

*Correspondence to: Wangxia Li, Blood Center of Wuhan, Wuhan, Hubei 430030, China. E-mail: xja33@163.com

Conflict of interest: The authors have declared no conflict of interests.

$\dagger$ The authors contributed equally to this work. sive. In this study, HLA-A, B, and DRB1 allele typing were performed using polymerase chain reactionsequence-specific oligonucleotide (PCR-SSOP), and polymerase chain reaction-sequencing based typing (PCR-SBT) techniques in 424 HIV-1/AIDS Han individuals from Hubei province in China. In this study, we investigated the correlation between HLA alleles and AIDS progression, and revealed the susceptibility/ resistance of individual HLA genes in association with HIV-1 infection and AIDS progression.

\section{MATERIALS AND METHODS}

\section{Object of study}

Four hundred and twenty-four HIV-1 virus seropositive individuals from the Han Chinese population in Hubei province were enrolled in this study. $\mathrm{CD} 4^{+} \mathrm{T}$ 
lymphocyte counts were monitored at the Wuhan Center for Disease Prevention and Control from January 2012 to August 2013. According to The HIV-1/ AIDS Diagnosis and Treatment Guidelines ${ }^{[10]}$, patients were classified into two groups: the AIDS group and HIV-1 infected group, according to the CD $4^{+} \mathrm{T}$ lymphocyte count and clinical symptoms. Two hundred and eleven individuals with AIDS symptoms, or those with less than 200 cells $/ \mathrm{mm}^{3} \mathrm{CD} 4^{+} \mathrm{T}$ lymphocytes were selected for the AIDS group and the remaining individuals were placed in the HIV-1 infected group.

Patients' basic information was collected and HLA$\mathrm{A}, \mathrm{B}$ and DRB1 allele typing (4-digit specificities) was performed with frozen $\left(-80^{\circ} \mathrm{C}\right)$ EDTA-anticoagulated whole blood samples from all 424 individuals. Informed consent from all subjects were obtained.

\section{Main reagents and instruments}

A Lambda Array Beads Multi-Analyte System for LAB Type or SSO Typing Test was bought from One Lambda, Inc. (USA). LIFECODES HLA-SSO Typing Kits were obtained from Tepnel Lifecodes Corporation (USA). SeCore SBT Kits were from Invitrogen (USA). PTC-225 Peltier Thermal Cycler was procured from MJ Research (USA). TIANamp Blood DNA Kit was from TIANGEN Biotech (China). DNA concentration detector was performed by Nano Drop 1000 Thermo Fisher Scientific (USA). A luminex 200 flow cytometry analyzer was bought from Luminex (USA). The ABI 3730 DNA sequencer was from Applied Biosystems (USA).

\section{The principle of interpretation}

According to the software interpretation and the HLA distribution of our cohort (taken from the Han population of Hubei province), we obtained 4 digit HLA genotyping results.

\section{Statistical analysis}

Allele frequencies, haplotype frequencies and Hardy-Weinberg equilibrium tests were analyzed and estimated using Arlequin Ver 3.0. Allele and haplotype distribution between the AIDS patients and HIV-1 infected group were compared with Epi Info 2002, the $P$ value was obtained by the $\chi^{2}$ test with Yates correction. The correlation between the HLA alleles and AIDS progression was analyzed by SPSS 18.0 with unconditional multivariate logistic regression analysis, so confounding factors such as age, sex and route of virus transmission were adjusted. The strength of association between HLA haplotype and AIDS progression was tested by means of an $\chi^{2}$ test using Epi Info 7. Values were set as; OR with a $95 \% \mathrm{CI}$, and $P<0.05$ was adopted as a significant difference. All statistical tests were two-sided tests. Alleles with an $\mathrm{OR}<1$ and an upper confidence limit of $95 \% \mathrm{CI}<1$ were considered resistant to HIV-1 infection: conversely, alleles with an OR>1 and a confidence limit lower than 95\% $\mathrm{CI}>1$ was considered susceptible to HIV-1 infection.

\section{RESULTS}

\section{Basic information}

We found that age and sex distributions between the two groups were of significant difference (Table 1). Within stratified sex groups, there were fewer AIDS patients in the female group $\left(87.2 \%\right.$ vs. $96.2 \%, \chi^{2}=11.44$, $P=0.000$ 7). Meanwhile, within stratified age groups, significant differences between AIDS patients and HIV-1 infected individuals were observed in the 18$29,50-59$ and $\geqslant 60$ age groups. There were more HIV-1 infected individuals in the 18-29 age group (39.9\% vs. $\left.14.7 \%, \chi^{2}=33.9, P<0.0001\right)$, while there were fewer in the $50-59$ age group (21.8\% vs. $10.3 \%$,

Table 1 Basic information of patients in AIDS patient group and HIV-1 infected group

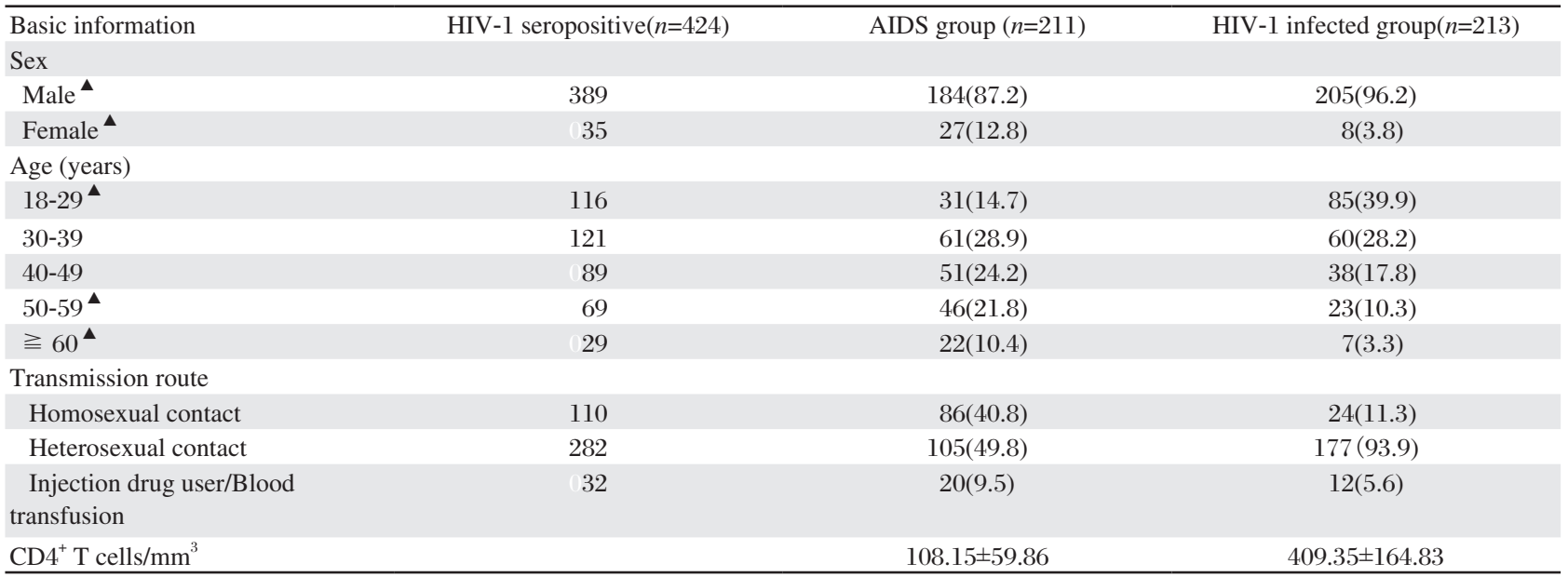


$\left.\chi^{2}=9.4, P=0.002\right)$ and $\geqslant 60$ age group $(10.4 \%$ vs $.3 .3 \%$, $\left.\chi^{2}=8.5, P=0.004\right)$. When stratified by transmission routes, there were more AIDS patients in the homosexual contact group $\left(40.8 \%\right.$ vs. $11.3 \%, \chi^{2}=52.9$, $P<0.0001)$, while there were fewer in the heterosexual contact group $\left(49.8 \%\right.$ vs. $\left.93.9 \%, \chi^{2}=48, P<0.0001\right)$. $\mathrm{CD} 4^{+} \mathrm{T}$ lymphocyte counts differed fairly significantly between AIDS patient and HIV-1 infected groups [(108.15 \pm 59.96$)$ cells $/ \mathrm{mm}^{3}$ vs. $(409.35 \pm 164.83)$ cells/ $\left.\mathrm{mm}^{3}, P<0.0001\right]$.

\section{Hardy-Weinberg equilibrium testing}

Exact tests of the Hardy-Weinberg equilibrium were performed using Arlequin Ver 3.0 (Table 2). Results indicated that the HLA-A, B and DRB1 allele frequencies observed in this cohort did not deviate from the Hardy-Weinberg equilibrium $(P>0.05)$, ensuring the reliability of our investigation data ${ }^{[11]}$.

Table 2 Hardy-Weinberg equilibrium tests of HLA genotyping in AIDS patient and HIV-1 infected groups

\begin{tabular}{lllll}
\hline Groups & Genetic locus & Observed & Expected & $P$ value \\
\hline AIDS patients $(n=211)$ & HLA-A & 0.86792 & 0.86276 & 0.31636 \\
& HLA-B & 0.94340 & 0.94225 & 0.10188 \\
& HLA-DRB1 & 0.91509 & 0.92154 & 0.00051 \\
HIV-1 infected $(n=213)$ & HLA-A & 0.90868 & 0.86493 & 0.00027 \\
& HLA-B & 0.94521 & 0.92380 & 0.92674 \\
& HLA-DRB1 & 0.91324 & 0.90175 & 0.87995 \\
\hline
\end{tabular}

Comparison of frequencies of HLA-A, B and DRB1 alleles in the AIDS patient and HIV-1 infected groups

A total of 26 HLA-A, 58 HLA-B and 37 HLADRB 1 alleles were identified from AIDS patient $(n=211)$ and HIV-1 infected $(n=213)$ groups. Among all 26 HLA-A alleles, no significant differences were observed between the AIDS patient and HIV-1 infected groups. However, in the AIDS patient group, the frequencies of the $\mathrm{B} * 57: 01$ allele $(0.0024 \mathrm{vs}$. $\left.0.0188, \chi^{2}=5.5, P=0.019\right)$ and DRB1*04:05 allele (0.035 5 vs. $\left.0.0728, \chi^{2}=6.1, P=0.014\right)$ were lower, while the DRB1*09:01 allele (0.234 6 vs. 0.1831 , $\left.\chi^{2}=3.8, P=0.05,\right)$ and $\mathrm{DRB} 1 * 01: 01$ allele $(0.0213$ vs. $\left.0.0023, \chi^{2}=5.5, P=0.019\right)$ were higher than those in the HIV-1 infected patient group. These differences were statistically significant, except for the DRB1*09:01 allele(Table 3).

Table 3 Frequencies ( $>1 \%$ ) of HLA-A, B and DRB1 alleles in the AIDS patient and HIV-1 infected groups

\begin{tabular}{|c|c|c|c|c|c|c|c|c|c|}
\hline \multirow[t]{2}{*}{ Alleles } & \multicolumn{2}{|c|}{$\begin{array}{c}\text { AIDS patients } \\
(2 \mathrm{n}=422)\end{array}$} & \multicolumn{2}{|c|}{$\begin{array}{c}\text { HIV-1 infected } \\
(2 \mathrm{n}=426)\end{array}$} & \multirow[t]{2}{*}{ Alleles } & \multicolumn{2}{|c|}{$\begin{array}{c}\text { AIDS patients } \\
(2 \mathrm{n}=422)\end{array}$} & \multicolumn{2}{|c|}{$\begin{array}{c}\text { HIV-1 infected } \\
(2 \mathrm{n}=426)\end{array}$} \\
\hline & Count & Frequency & Count & Frequency & & Count & Frequency & Count & Frequency \\
\hline$A^{*} 11: 01$ & 111 & 0.2630 & 121 & 0.2840 & B*55:02 & 11 & 0.0261 & 15 & 0.0352 \\
\hline$A * 24: 02$ & 68 & 0.1564 & 61 & 0.1432 & $B * 40: 06$ & 9 & 0.0213 & 9 & 0.0211 \\
\hline$A * 02: 07$ & 61 & 0.1445 & 58 & 0.1362 & B*39:01 & 8 & 0.0190 & 11 & 0.0258 \\
\hline$A * 02: 01$ & 43 & 0.1019 & 33 & 0.0775 & B*52:01 & 8 & 0.0190 & 12 & 0.0282 \\
\hline A*33:03 & 24 & 0.0569 & 31 & 0.0728 & B*40:02 & 7 & 0.0166 & 13 & 0.0305 \\
\hline$A * 02: 03$ & 22 & 0.0521 & 17 & 0.0399 & $\mathrm{~B} * 57: 01^{\mathbf{\Lambda}}$ & 1 & 0.0024 & 8 & 0.0188 \\
\hline$A * 31: 01$ & 19 & 0.0450 & 16 & 0.0376 & $\mathrm{~B} * 15: 18$ & 0 & 0 & 5 & 0.0117 \\
\hline$A * 30: 01$ & 15 & 0.0355 & 19 & 0.0446 & DRB1*09:01 & 99 & 0.2346 & 78 & 0.1831 \\
\hline$A * 01: 01$ & 12 & 0.0284 & 10 & 0.0235 & DRB1*12:02 & 52 & 0.1232 & 38 & 0.0892 \\
\hline$A * 26: 01$ & 12 & 0.0284 & 14 & 0.0329 & DRB1*15:01 & 37 & 0.0877 & 31 & 0.0728 \\
\hline$A * 11: 02$ & 10 & 0.0237 & 11 & 0.0258 & DRB1*08:03 & 35 & 0.0829 & 46 & 0.1080 \\
\hline$A * 02: 06$ & 12 & 0.0284 & 10 & 0.0235 & DRB $1 * 07: 01$ & 25 & 0.0592 & 23 & 0.0540 \\
\hline$A * 03: 01$ & 4 & 0.0095 & 7 & 0.0164 & DRB1*03:01 & 23 & 0.0545 & 24 & 0.0563 \\
\hline$B * 46: 01$ & 79 & 0.1872 & 63 & 0.1479 & DRB1*11:01 & 21 & 0.0498 & 21 & 0.0493 \\
\hline$B * 40: 01$ & 61 & 0.1445 & 48 & 0.1127 & DRB1*14:01 & 16 & 0.0379 & 13 & 0.0305 \\
\hline$B * 13: 01$ & 38 & 0.0900 & 26 & 0.0610 & DRB1 $* 04: 05^{\boldsymbol{\Lambda}}$ & 31 & 0.0735 & 15 & 0.0352 \\
\hline$B * 58: 01$ & 23 & 0.0545 & 22 & 0.0516 & DRB1*04:06 & 14 & 0.0332 & 12 & 0.0282 \\
\hline$B * 15: 01$ & 22 & 0.0521 & 17 & 0.0399 & DRB1*12:01 & 12 & 0.0308 & 11 & 0.0258 \\
\hline$B * 15: 02$ & 20 & 0.0474 & 21 & 0.0493 & DRB1*14:05 & 12 & 0.0284 & 8 & 0.0188 \\
\hline$B * 51: 01$ & 19 & 0.0450 & 19 & 0.0446 & DRB1*16:02 & 11 & 0.0261 & 14 & 0.0329 \\
\hline$B * 54: 01$ & 15 & 0.0355 & 18 & 0.0423 & DRB1*13:02 & 9 & 0.0213 & 10 & 0.0235 \\
\hline B*13:02 & 13 & 0.0308 & 19 & 0.0023 & DRB $1 * 15: 02$ & 8 & 0.0190 & 13 & 0.0305 \\
\hline B*35:01 & 12 & 0.0284 & 10 & 0.0235 & DRB1*04:03 & 7 & 0.0166 & 7 & 0.0164 \\
\hline B*38:02 & 11 & 0.0261 & 14 & 0.0329 & DRB1 $* 01: 01{ }^{\wedge}$ & 9 & 0.0213 & 1 & 0.0023 \\
\hline
\end{tabular}


Two-locus of HLA-A-B, HLA-A-DRB1, HLA-BDRB 1 and 3-locus of HLA-A-B-DRB1 haplotypes with frequencies of more than $1 \%$ were estimated and compared between two groups using Arlequin Ver.3.0 (Table 4). A total of 140 HLA-A-B, 125 HLA-ADRB1, 159 HLA-B-DRB1, and 240 HLA-A-B-DRB1 haplotypes were analyzed in the AIDS patient group. Simultaneously, a total of 143 HLA-A-B, 139 HLAA-DRB1, 184 HLA-B-DRB1 and 258 HLA-A-BDRBI haplotypes were analyzed in HIV-1 infected group.

For HLA-A-B-HLA-B-DRB 1 and HLA-A-
DRB1 haplotypes, $\mathrm{A} * 11: 01-\mathrm{B} * 52: 01$ (0 vs. 0.0164, $P=0.024), \mathrm{A} * 11: 01-\mathrm{B} * 55: 02(0.0024$ vs. 0.0237 , $P=0.016$ ), $\mathrm{A}^{*} 24: 02-\mathrm{B} * 13: 01$ (0 vs 0.021 1, $P=0.008$ ), $\mathrm{A}^{*} 02: 01-\mathrm{DRB1}{ }^{*} 08: 03$ (0 vs. 0.014 1, $P=0.042$ ), and A*11:01-DRB1*08:03 (0.011 8 vs. 0.039 9, $P=0.02)$ were significantly underrepresented, while $A * 02: 01-$ DRB 1*12:02 (0.0213 vs. 0, $P=0.007)$ was significantly overrepresented in the AIDS patient group. For HLA-A-B-DRB 1 haplotypes, a quite common haplotype A*02:07-B*13:01-DRB1*12:02(0.014 2 vs. $0, P=0.039)$ was significantly overrepresented in the AIDS patient group.

Table 4 HLA haplotype frequencies in AIDS patient and HIV-1 infected groups

\begin{tabular}{|c|c|c|c|c|c|c|c|c|}
\hline \multirow[b]{2}{*}{$\begin{array}{l}\text { Haplotype } \\
\text { (2-locus) }\end{array}$} & \multicolumn{2}{|c|}{ Frequency } & \multirow[b]{2}{*}{$\begin{array}{l}\text { Haplotype } \\
\text { (2-locus) }\end{array}$} & \multicolumn{2}{|c|}{ Frequency } & \multirow[b]{2}{*}{$\begin{array}{l}\text { Haplotype } \\
\text { (2-locus) }\end{array}$} & \multicolumn{2}{|c|}{ Frequency } \\
\hline & $\begin{array}{l}\text { AIDS } \\
\text { patients } \\
(2 n=422)\end{array}$ & $\begin{array}{l}\text { HIV-1 } \\
\text { infected } \\
(2 n=426)\end{array}$ & & $\begin{array}{l}\text { AIDS } \\
\text { patients } \\
(2 n=422)\end{array}$ & $\begin{array}{l}\text { HIV-1 } \\
\text { infected } \\
(2 n=426)\end{array}$ & & $\begin{array}{l}\text { AIDS } \\
\text { patients } \\
(2 n=422)\end{array}$ & $\begin{array}{l}\text { HIV-1 } \\
\text { infected } \\
(2 n=426)\end{array}$ \\
\hline$A * 02: 07-B * 46: 01$ & 0.1084 & 0.1033 & A*02:07-DRB1*09:01 & 0.0640 & 0.0563 & B*46:01-DRB1*09:01 & 0.0995 & 0.0798 \\
\hline$A * 11: 01-B * 40: 01$ & 0.0664 & 0.0610 & A*11:01-DRB1*09:01 & 0.0521 & 0.0305 & B*13:01-DRB1*12:02 & 0.0379 & 0.0117 \\
\hline A*11:01-B*13:01 & 0.0498 & 0.0282 & A*24:02-DRB1*09:01 & 0.0450 & 0.0352 & B*58:01-DRB1*03:01 & 0.0379 & 0.0352 \\
\hline$A * 24: 02-B * 40: 01$ & 0.0379 & 0.0188 & A*11:01-DRB1*15:01 & 0.0427 & 0.0329 & B*40:01-DRB1*09:01 & 0.0332 & 0.0117 \\
\hline$A * 33: 03-B * 58: 01$ & 0.0355 & 0.0423 & A*11:01-DRB1*12:02 & 0.0403 & 0.0446 & $\mathrm{~B} * 13: 02-\mathrm{DRB} 1 * 07: 01$ & 0.0284 & 0.0258 \\
\hline A*11:01-B*15:02 & 0.0308 & 0.0352 & A*02:07-DRB1*08:03 & 0.0332 & 0.0258 & $\mathrm{~B} * 46: 01-\mathrm{DRB} 1 * 08: 03$ & 0.0284 & 0.0258 \\
\hline$A^{*} 30: 01-B * 13: 02$ & 0.0308 & 0.0399 & A*30:01-DRB1*07:01 & 0.0308 & 0.0235 & $\mathrm{~B} * 15: 02-\mathrm{DRB} 1 * 12: 02$ & 0.0261 & 0.0282 \\
\hline $\mathrm{A}^{*} 11: 01-\mathrm{B} * 15: 01$ & 0.0261 & 0.0164 & A*31:01-DRB1*09:01 & 0.0284 & 0.0094 & B*13:01-DRB1*15:01 & 0.0237 & 0.0164 \\
\hline A*02:03-B*38:02 & 0.0237 & 0.0164 & A*33:03-DRB1*03:01 & 0.028 & 0.0305 & $\mathrm{~B} * 40: 01-\mathrm{DRB1} * 12: 02^{\Delta}$ & 0.0237 & 0 \\
\hline$A * 11: 01-B * 46: 01$ & 0.0213 & 0.0188 & A*02:01-DRB1*12:02 & 0.0213 & 0 & B*51:01-DRB1*09:01 & 0.0237 & 0.0141 \\
\hline$A * 02: 07-B * 40: 01$ & 0.0142 & 0.0023 & A*02:01-DRB1*15:01 & 0.0190 & 0.0047 & B*40:01-DRB1*15:01 & 0.0166 & 0.0094 \\
\hline A*02:01-B*13:01 & 0.0095 & 0 & A*11:01-DRB1*11:01 & 0.0190 & 0.0141 & $\mathrm{~B} * 15: 01-\mathrm{DRB} 1 * 09: 01$ & 0.0142 & 0.0094 \\
\hline $\mathrm{A} * 02: 07-\mathrm{B} * 13: 01$ & 0.0024 & 0 & A*02:07-DRB1*12:02 & 0.0166 & 0.0117 & $\mathrm{~B} * 40: 06-\mathrm{DRB} 1 * 09: 01$ & 0.0142 & 0.0070 \\
\hline$A * 11: 01-B * 51: 01$ & 0.0142 & 0.0094 & $\mathrm{~A}^{*} 11: 01-\mathrm{DRB} 1 * 14: 01$ & 0.0166 & 0.0070 & B*54:01-DRB1*04:05 & 0.0142 & 0.0188 \\
\hline$A * 24: 02-B * 40: 02$ & 0.0047 & 0.0188 & A*11:01-DRB1*16:02 & 0.0166 & 0.0070 & $\mathrm{~B} * 15: 01-\mathrm{DRB} 1 * 04: 06$ & 0.0118 & 0.0070 \\
\hline A*11:01-B*54:01 & 0.0142 & 0.0117 & A*02:01-DRB1*09:01 & 0.0142 & 0.0141 & B*40:01-DRB1*11:01 & 0.0118 & 0.0070 \\
\hline $\mathrm{A}^{*} 11: 01-\mathrm{B}^{*} 55: 02^{\mathbf{\Delta}}$ & 0.0024 & 0.0237 & A*11:01-DRB1*04:05 & 0.0118 & 0.0282 & $\mathrm{~B} * 46: 01-\mathrm{DRB} 1 * 04: 05$ & 0.0118 & 0 \\
\hline $\mathrm{A}^{*} 11: 01-\mathrm{B} * 52: 01^{\boldsymbol{\Delta}}$ & 0 & 0.0164 & A*11:01-DRB1*08:03 & 0.0118 & 0.0399 & B*08:01-DRB1*03:01 & 0.0095 & 0.0094 \\
\hline $\mathrm{A}^{*} 24: 02-\mathrm{B} * 13: 01^{\boldsymbol{\Lambda}}$ & 0 & 0.0211 & $\mathrm{~A}^{*} 02: 01-\mathrm{DRB1}{ }^{*} 08: 03^{\boldsymbol{\Lambda}}$ & 0 & 0.0141 & $\mathrm{~B} * 15: 01-\mathrm{DRB} 1 * 15: 01$ & 0.0095 & 0.0047 \\
\hline \multirow[b]{2}{*}{$\begin{array}{l}\text { Haplotype } \\
\text { (3-locus) }\end{array}$} & \multicolumn{2}{|c|}{ Frequency } & \multirow[b]{2}{*}{$\begin{array}{l}\text { Haplotype } \\
\text { (3-locus) }\end{array}$} & \multicolumn{2}{|c|}{ Frequency } & \multirow{2}{*}{$\begin{array}{c}\text { Haplotype } \\
\text { (3-locus) }\end{array}$} & \multicolumn{2}{|c|}{ Frequency } \\
\hline & $\begin{array}{l}\text { AIDS } \\
\text { patients } \\
(2 n=422) \\
\end{array}$ & $\begin{array}{l}\text { HIV-1 } \\
\text { infected } \\
(2 n=426)\end{array}$ & & $\begin{array}{l}\text { AIDS } \\
\text { patients } \\
(2 n=422) \\
\end{array}$ & $\begin{array}{l}\text { HIV-1 } \\
\text { infected } \\
(2 n=426) \\
\end{array}$ & & $\begin{array}{l}\text { AIDS } \\
\text { patients } \\
(2 n=422)\end{array}$ & $\begin{array}{l}\text { HIV-1 } \\
\text { infected } \\
(2 n=426)\end{array}$ \\
\hline $\begin{array}{l}\text { A*33:03-B*58:01- } \\
\text { DRB1*03:01 }\end{array}$ & 0.0308 & 0.0282 & $\begin{array}{c}\text { A*11:01-B*15:02- } \\
\text { DRB1*12:02 }\end{array}$ & 0.0142 & 0.0023 & $\begin{array}{c}\text { A*11:01-B*51:01- } \\
\text { DRB1*09:01 }\end{array}$ & 0.0095 & 0.0047 \\
\hline $\begin{array}{l}\text { A*30:01-B*13:02- } \\
\text { DRB1*07:01 }\end{array}$ & 0.0284 & 0.0211 & $\begin{array}{c}\text { A*11:01-B*46:01- } \\
\text { DRB1*09:01 }\end{array}$ & 0.0142 & 0 & $\begin{array}{c}\text { A*11:01-B*54:01- } \\
\text { DRB1*09:01 }\end{array}$ & 0.0095 & 0 \\
\hline $\begin{array}{l}\text { A*02:07-B*46:01- } \\
\text { DRB1*08:03 }\end{array}$ & 0.0237 & 0.0211 & $\begin{array}{c}\mathrm{A} * 24: 02-\mathrm{B} * 40: 01- \\
\text { DRB1*09:01 }\end{array}$ & 0.0142 & 0.0258 & $\begin{array}{c}\mathrm{A} * 24: 02-\mathrm{B} * 40: 01- \\
\mathrm{DRB} 1 * 12: 02\end{array}$ & 0.0095 & 0 \\
\hline $\begin{array}{l}\text { A*11:01-B*13:01- } \\
\text { DRB1*15:01 }\end{array}$ & 0.0237 & 0.0094 & $\begin{array}{c}\text { A*02:01-B*40:01- } \\
\text { DRB1*09:01 }\end{array}$ & 0.0118 & 0.0188 & $\begin{array}{c}\text { A*01:01-B*52:01- } \\
\text { DRB1*15:02 }\end{array}$ & 0.0071 & 0.0023 \\
\hline $\begin{array}{l}\text { A*11:01-B*40:01- } \\
\text { DRB1*08:03 }\end{array}$ & 0.0190 & 0.0352 & $\begin{array}{c}\text { A*11:01-B*13:01- } \\
\text { DRB1*12:02 }\end{array}$ & 0.0095 & 0 & $\begin{array}{c}\text { A*24:02-B*46:01- } \\
\text { DRB1*09:01 }\end{array}$ & 0.0166 & 0.0023 \\
\hline $\begin{array}{l}\text { A*11:01-B*40:01- } \\
\text { DRB1*12:02 }\end{array}$ & 0.0166 & 0.0095 & $\begin{array}{c}\text { A*11:01-B*15:01- } \\
\text { DRB1*04:06 }\end{array}$ & 0.0095 & 0.0047 & & & \\
\hline
\end{tabular}


The multivariate logistic regression analysis using the forward stepwise method was conducted for all alleles listed in Table 1 respectively, to analyze the association of HLA-A, B, and DRBl alleles with HIV-1 infection between the AIDS patient and HIV-1 infected groups (Table 5).
Overall, our investigation revealed that all HLAA alleles had no effect on HIV-1 infection, as for all HLA-B and HLA-DRB1 alleles, our results showed that DRB1*04:05 was a risk factor for HIV-1 infection, while individuals with $\mathrm{B} * 57: 01$ allele were more likely to be resistant to AIDS progression in the $\mathrm{Wu}$ -

Table 5 Binary logistic regression analysis

\begin{tabular}{|c|c|c|c|c|c|c|}
\hline & \multirow{2}{*}{ Logistic regression analysis } & & \multirow{2}{*}{ Sig } & \multirow{2}{*}{$\operatorname{Exp}(B)$} & \multicolumn{2}{|c|}{$95 \%$ CI.for $\operatorname{Exp}(B)$} \\
\hline & & & & & Lower & Upper \\
\hline \multirow[t]{3}{*}{1} & Step2 & $\mathrm{B} * 15: 18$ & 0.999 & $1.647 \mathrm{E} 9$ & 0 & - \\
\hline & & $\mathrm{B} * 57: 01$ & 0.000 & 0.154 & 0.083 & 0.287 \\
\hline & & Constant & 0.782 & 0.981 & & \\
\hline \multirow[t]{2}{*}{2} & Step2 & $\mathrm{DRB1} * 01: 01$ & 0.063 & 7.325 & 0.897 & 59.809 \\
\hline & & $\mathrm{DRB1} 1 * 04: 05$ & 0.017 & 2.163 & 1.149 & 4.068 \\
\hline \multirow[t]{4}{*}{3} & Step3 & Sex:Male & 0.001 & 1.984 & 1.344 & 2.927 \\
\hline & & Age & 0.013 & 0.337 & 0.142 & 0.798 \\
\hline & & Transmission route & 0.000 & 0.957 & 0.940 & 0.973 \\
\hline & & Constant & 0.020 & 5.235 & & \\
\hline \multirow[t]{4}{*}{4} & Step $5^{\mathrm{a}}$ & $B * 57: 01$ & 0.003 & 0.326 & 0.157 & 0.678 \\
\hline & & Sex:Male & 0.001 & 2.909 & 1.579 & 5.359 \\
\hline & & Age & 0.000 & 0.956 & 0.944 & 0.968 \\
\hline & & Constant & 0.284 & 0.600 & & \\
\hline \multirow[t]{6}{*}{5} & Step $4^{\mathrm{a}}$ & DRB1*04:05 & 0.024 & 2.183 & 1.109 & 4.296 \\
\hline & & $\mathrm{DRB1} * 04: 07$ & 0.999 & $2.682 \mathrm{E} 9$ & 0.000 & - \\
\hline & & Sex:Male & 0.000 & 3.152 & 1.689 & 5.884 \\
\hline & & Age & 0.000 & 0.956 & 0.944 & 0.967 \\
\hline & & Transmission route & 0.000 & 1.930 & 1.462 & 2.548 \\
\hline & & Constant & 0.263 & 0.584 & & \\
\hline
\end{tabular}

Multivariate logistic regression analysis: Covariates (all HLA-A,B and DRB1 alleles in Table 3 were entered independently); Method: Forward (Wald); Probability for stepwise = Entry (0.05), Remove (0.1). a:After eliminate the influence of transmission route, sex and age.

han Han population, even after eliminating the influence of transmission route, sex and age. In addition, our investigation revealed that sex, age and transmission routes of the hosts may affect HIV-l progression, female and homosexual contact were risk factors for HIV-1 infection, while the age of 18 to 29 showed some protective effects to HIV-1 infection.

To explore the correlation between HLA haplotypes and AIDS progression, we compared those 2- and 3-locus haplotypes with significantly different frequencies $(P<0.05)$ between the two comparison groups $($ Table 4$)$. Epi Info 7.0 was used to calculate their corrected $P$ values, OR values and $95 \%$ CI values ${ }^{[12]}$. As shown in Table 6, individuals with $\mathrm{A}^{*} 02: 01-\mathrm{DRB} 1 * 12: 02$, $\mathrm{B} * 13: 01-\mathrm{DRB} 1 * 12: 02, \mathrm{~B} * 40: 01-\mathrm{DRB} 1 * 12: 02$ and $A * 02: 07-B * 13: 01-D R B 1 * 12: 02$ haplotypes were of great susceptibility to the HIV-1 infection, while those with $A * 11: 01-B * 52: 01, A * 11: 01-B * 55: 02$, $A * 24: 02-B * 13: 01, A * 02: 01-D R B 1 * 08: 03, A * 11: 01-$ DRB1*08:031 haplotypes are more likely to be resistant to AIDS progression. In addition, we also compared the HLA-A, B, DRB1 homozygotes and HLABw4, Bw6 serological types in these two groups. Our results suggested that the homozygosity of A-A, B-B, DRB1-DRB1 and the serotype group of Bw4-Bw4, Bw4-Bw6, Bw6-Bw6 were not significantly different (Table 6).

\section{DISCUSSION}

In order to investigate the associations between HLA alleles and susceptibility or resistance to the HIV-1 infection in the Han Chinese population in Hubei province, we analyzed and compared the distributions of HLA-A, B, DRBI alleles, frequencies of HLA-A-B, HLA-B-DRB1, HLA-A-DRB1, HLA-A$\mathrm{B}-\mathrm{DRB} 1$ haplotypes, and the HLA-A, B, DRB1 homozygotes and HLA-Bw4, Bw6 serological types in these two groups respectively. Our results showed that AIDS progression was significantly associated with a lower frequency of $\mathrm{B} * 57: 01$ and a high frequency of DRB1*04:05 alleles, but not with the homozygosity of A-A, B-B, DRB1-DRB1, or the serotype group of Bw4-Bw4, Bw4-Bw6, Bw6-Bw6.

HLA class I antigens are endogenous antigenpresenting molecules. Their antigen specificity is 
108 Prognostic and predictive value of IGFBP-6 in head and neck squamous cell carcinoma, 2018, 2(2)

Table 6 Comparison of HLA haplotypes and genotypes in AIDS patient and HIV-1 infected groups

\begin{tabular}{|c|c|c|c|c|c|c|}
\hline Haplotype/Genotype & AIDS patients $[n(\%)]$ & HIV-1 infected $[n(\%)]$ & Odds ratios & $95 \% \mathrm{CI}$ & $P$ value & Corrected $P$ value \\
\hline \multicolumn{7}{|l|}{ 2-locus Haplotype } \\
\hline $\mathrm{A} * 11: 01-\mathrm{B} * 52: 01$ & $0(0)$ & $7(1.6)$ & 0.00 & $0.00-0.72$ & 0.008 & 0.024 \\
\hline $\mathrm{A} * 11: 01-\mathrm{B} * 55: 02$ & $1(0.2)$ & $10(2.3)$ & 0.099 & $0.013-0.775$ & 0.007 & 0.016 \\
\hline $\mathrm{A} * 24: 02-\mathrm{B} * 13: 01{ }^{\boldsymbol{\Delta}}$ & $0(0)$ & $9(2.1)$ & 0.00 & $0.00-0.12$ & 0.003 & 0.008 \\
\hline A*02:01-DRB1 $* 08: 03^{\Delta}$ & $0(0)$ & $6(1.4)$ & 0.00 & $0.00-0.70$ & 0.014 & 0.042 \\
\hline $\mathrm{A}^{*} 02: 01-\mathrm{DRB1}{ }^{*} 12: 02^{\boldsymbol{\Delta}}$ & $9(2.1)$ & $0(0)$ & NA & & 0.002 & 0.007 \\
\hline A*11:01-DRB1*08:03 & $5(1.2)$ & $17(4.0)$ & 0.29 & $0.11-0.79$ & 0.010 & 0.020 \\
\hline B*13:01-DRB1*12:02 & $16(3.8)$ & $5(1.2)$ & 3.3 & $1.2-9.1$ & 0.014 & 0.026 \\
\hline B*40:01-DRB1*12:02 & $10(2.4)$ & $0(0.0)$ & NA & & 0.001 & 0.004 \\
\hline \multicolumn{7}{|l|}{ 3-locus Haplotype } \\
\hline $\mathrm{A} * 02: 07-\mathrm{B} * 13: 01-\mathrm{DRB} 1 * 12: 02$ & $6(1.4)$ & $0(0.0)$ & NA & & 0.014 & 0.039 \\
\hline \multicolumn{7}{|l|}{ genetype } \\
\hline Bw4-Bw4 & $28(6.6)$ & $23(5.4)$ & 1.26 & $0.68-2.37$ & 0.43 & \\
\hline Bw4-Bw6 & $88(20.9)$ & 102(23.9) & 0.78 & $0.52-1.16$ & 0.20 & \\
\hline Bw6-Bw6 & $95(22.5)$ & $88(20.7)$ & 1.16 & $0.89-1.31$ & 0.44 & \\
\hline A-A & $41(9.7)$ & $44(10.3)$ & 0.93 & $0.58-1.49$ & 0.75 & \\
\hline B-B & 15(3.6) & $18(4.2)$ & 0.83 & $0.41-1.69$ & 0.61 & \\
\hline DRB1-DRB1 & $20(4.7)$ & $21(4.9)$ & 0.96 & $0.50-1.80$ & 0.89 & \\
\hline
\end{tabular}

$\boldsymbol{\Delta}$ : corrected $P$ value $<0.05$ of comparative distribution of haplotypes and genotypes in AIDS patient and HIV-1 infected groups. NA not available

dependent on their alpha heavy chains, which are encoded by HLA-A, B, C loci. Gene polymorphism can influence HIV-1 antigen-presentation and the ability of effector T cells to HIV-1, thus was significantly associated with HIV-1 virus transmission, AIDS progression, opportunistic HIV-l infection, antiviral therapy and vaccine efficacy. The CTL's ability to viral protein epitope varies according to their immunogenicity listed in an order of Nef $>$ Gag $>$ Env $>$ Vif $>$ Rev $>$ Vpr $>$ Tat $>$ Vpu. The Gag protein epitope was mainly presented by HLA-I antigens ${ }^{[13]}$.

\section{HLA-A}

The HLA-A*02 allele was the most widely distributed allele and played an important role in host's resistance to HIV-1 infection ${ }^{[14]}$. The ninth residue of the HLA-A * 02 allele coded protein was aromatic AA phenylalanine or tyrosine. Their narrow B hollows influence the specificity of $\mathrm{F}$ hollows to peptide antigen. This results in antigen peptide presented by HLA molecule hard to be recognized by immune cells, therefore increases patients' susceptibility to HIV-1. Thus, HLA-A*02 alleles were of great significance to the reasonably design of HIV-1 vaccines ${ }^{[15]}$. We previously reported that $A^{*} 02: 06$ was a risk factor of HIV-1 infection ${ }^{[14]}$. In this study we found that besides $\mathrm{A} * 0206$, the frequencies of $\mathrm{A} * 02: 01$ and $\mathrm{A} * 02: 03$ were also higher in the AIDS patient group than those in the HIV-1 infected group. However, the differences were not statistically significant, which might be due to lower case numbers in each allele group.

\section{HLA-B}

HLA-B alleles can exert greater immune selec- tion pressure on HIV-1 than HLA-A alleles, and significantly influence the HIV-1 set-point and $\mathrm{CD} 4^{+} \mathrm{T}$ lymphocyte count of an infected person, thus affecting AIDS progression. The polymorphism of HLA-B alleles was the most complex of all HLA-I molecules and was closely related to virus control. It has been reported that individuals who have the HLA-B*57 allele showed increased CTL responses to Gag-mutated hypoallergenic $\mathrm{HIV}-1^{[16]}$. In addition, HLA-B*57:01 allele was closely associated with Abacavir Hypersensitivity symdrome (ASH) because Abacavir can induce the HLA-B*57:01-restricted $\mathrm{CD}^{+}{ }^{+} \mathrm{T}$ cell immune response. Thus, screening for HLA-B*57:01 before the administering of Abacavir to patients was an essential step recommended by both the United States and Europe in HIV treatment guidelines ${ }^{[17]}$. Our observation seems to support previous findings. We found a significantly lower frequency of $B^{*} 57: 01$ in the AIDS group, as compared to that in the HIV1 infected group, which implied that HLA-B*57:01 mediated $\mathrm{CD}^{+} \mathrm{T}$ lymphocyte immunity may play an important role in preventing the process of HIV-1 infection to AIDS progression. Therefore, HIV-infected individuals with $\mathrm{B}^{*} 57: 01$ allele are less likely to progress to AIDS.

\section{HLA-DRB1}

$\mathrm{CD} 4^{+} \mathrm{T}$ lymphocytes' antigen epitopes were mainly presented by HLA- II molecules, which could induce a protective $\mathrm{CD} 4^{+} \mathrm{T}$ lymphocyte immune response and prevent virus infection. HLA-DRB1 lays an important role in organism antiviral immune response mediated by $\mathrm{CD} 4^{+} \mathrm{T}$ lymphocyte. An early study in 1999 by Aikawa concluded that HLA-DRB1*04:05 
allele promoted HCV infection into cirrhosis ${ }^{[18]}$. Research results from Li et al. ${ }^{[19]}$ also indicated that HLA-DRB 1*04:05 was responsible for Hepatitis $B$ induced cirrhosis. Our study revealed that HLA$\mathrm{DRB1} * 04: 05$ has an association with susceptibility to HIV-1 infection, but the mechanism remains unclear. $\mathrm{DRB} 1 * 04: 05$ is a dominated gene in the Han population of Hubei province, according to the frequency dependent selection principle, which led us to assume that HIV-1 has been remarkably adaptive to widely distributed DRB1*04:05, and the human body was unable to protect itself from HIV-1 infection.

\section{Common haplotypes}

Our results showed that most haplotypes were of higher frequency in the AIDS patient group than in the HIV-1 infected group, and high-frequency haplotypes were risk factors for AIDS progression, which reconfirmed the principle of HLA frequency-dependent selection, i.e. the virus mutated for escaping from an effective immune response mediated by common haplotypes ${ }^{[20]}$.

\section{HLA homozygous allele and Bw4/Bw6 gene}

Our results indicated that HLA-A, B, DRB1 homozygotes and HLA-Bw4, Bw6 serological types had no association with AIDS progression, which was not in agreement with current research conclusions. Regional and racial differences could have caused this deviation. There were much limitation in our research because we could not obtain the exact times of HIV-1 infection, and time bias may occurred in the process of estimating the disease course. It suggested that a cohort study will be more meaningful in the future.

Other host factors such as age, sex and host transmission route showed significant differences between these two groups. Our research indicated that: (1) Being female was an AIDS risk factor, due to female physiology; (2) The 18 to 29 years old age group showed some resistance to AIDS, mainly because younger hosts have stronger immunity and greater resistance to viral infection; (3) Homosexual contact was a risk factor of AIDS. It has been reported that the number of AIDS infections are rising in the Chinese MSM (men who have sex with men) population yearly, so we recommend that this specific group has priority for both intervention and testing ${ }^{[21-23]}$. Meanwhile, multifactor logistic regression analysis in this study also confirmed that the host's sex, age and transmission route were associated with AIDS progression independently.

In conclusion, this observational study revealed that HLA gene polymorphism is associated with HIV-1 infection and AIDS progression. Different HLA genes seem to be associated with either the host's resistance (B*5701) or susceptibility (DRB1*0405) to HIV-1 infection or AIDS progression. This report serves as a good basis for further investigations regarding the molecular mechanisms involved in these associations.

\section{Acknowledgements}

This work was supported by grants from the funding of Wuhan Health Planning Commission (No. WX17Q34), and the Natural Science Funds of Hubei Province (No. 2017CFB559), and the Foundation of Young Medical Talents in Wuhan, China.

\section{References}

[1] Langford SE, Ananworanich J, Cooper DA. Predictors of disease progression in HIV-1 infection:a review[J]. AIDS Res Ther, 2007, 4(1):11

[2] Kelleher AD, Long C, Holmes EC, et al. Clustered mutations in HIV-1 gag are consistently required for escape from HLA-B27-restricted cytotoxic T lymphocyte responses[J]. J Exp Med, 2001, 193(3):375-386

[3] Honeyborne I, Prendergast A, Pereyra F, et al. Control of human immunodeficiency virus type 1 is associated with HLA-B*13 and targeting of multiple gag-specific CD8+ T-cell epitopes[J]. J Virol, 2007, 81(7):3667-3672

[4] Pelak K, Goldstein DB, Walley NM, et al. Host determinants of HIV-1 control in African Americans[J]. $J$ Infect Dis, 2010, 201(8):1141-1149

[5] Kawashima Y, Kuse N, Gatanaga H, et al. Long-term control of HIV-1 in hemophiliacs carrying slowprogressing allele HLA-B*5101[J]. JVirol, 2010, 84(14):7151-7160

[6] Jia MM, Hong KX, Ren GL, et al.Correlation study of HLA class I alleles polymorphism and viral load in central China's infected People[J].Chin J Microbiol.Immunol,2010,30(5):438-442

[7] Huang X, Ling H, Feng L, et al. Human leukocyte antigen profile in HIV-1 infected individuals and AIDS patients from Chongqing, China[J]. Microbiol Immunol, 2009, 53(9):512-523

[8] Ye L, LEI Yong ling CHEN Sha Bin.Study on polymorphisms of HLA-A,HLA-B and HLA-DRB1 loci among HIV-1 long-term nonprogressors in Lishui[J]. Chin J AIDS STD, 2015, 21(8):656-659

[9] Zhang X, Wang Y, Wang S, et al. The influence of human leucocyte antigen- I polymorphisms on plasma viral load in human immunodeficiency virus infected male homosexual population in Beijing[J]. Zhonghua Chuan Ran Bing Za Zhi(in Chinese), 2013, 31(3):173178

[10] AIDS Group. Society of infection diseases, Chinese medical association[J]. Chin.J Clin.Infect.Dis, 2015, 8(5):385401

[11] Excoffier L, Laval G, Schneider S. Arlequin (version 3.0): an integrated software package for population ge- 
110 Prognostic and predictive value of IGFBP-6 in head and neck squamous cell carcinoma, 2018, 2(2)

netics data analysis[J]. Evol Bioinform Online, 2005, 1(1):47-50

[12] Yan WL. Genome-wide association study on complex diseases: genetic statistical issues[J]. Yi Huan, 2008, 30(5):543-549

[13] Masemola A, Mashishi T, Khoury G, et al. Hierarchical targeting of subtype $\mathrm{C}$ human immunodeficiency virus type 1 proteins by $\mathrm{CD}^{+} \mathrm{T}$ cells: correlation with viral load[J]. J Virol, 2004, 78(7):3233-3243

[14] Li WX, Xia JA, Zhou X, et al. Association of HLA alleles (A, B, DRB1) and HIV-1 infection in the Han population of Hubei, China[J]. Journal of Huazhong University of Science and Technology - Medical Sciences, (in chinese)2017, 37(1):131-139

[15] Nielsen M, Justesen S, Lund O, et al. NetMHC- II pan2.0-Improved pan-specific HLA-DR predictions using a novel concurrent alignment and weight optimization training procedure[J]. Immunome Res, 2010, 6(1):9

[16] Miura T, Brockman MA, Schneidewind A, et al. HLAB57/B*5801 human immunodeficiency virus type 1 elite controllers select for rare gag variants associated with reduced viral replication capacity and strong cytotoxic T-lymphocyte [corrected] recognition[J]. J Virol, 2009, 83(6):2743-2755

[17] Department of Health and Human Services. Panel on
Antiretroviral Guidelines for Adults and Adolescents. Guidelines for the Use of Antiretroviral Agents in Adults and Adolescents Living with HIV[2]. Available at http:// www.aidsinfo.nih.gov/ContentFiles/ AdultandAdolescentGL.pdf.

[18] Aikawa T, Kojima M, Onishi H, et al. HLA DRB1 and DQB1 alleles and haplotypes influencing the progression of hepatitis C[J]. J Med Virol, 1996, 49(4):274-278

[19] Li DL, Jz P, Zhao SM, et al. Correlation study of hepatitis cirrhosis and HLA-DRB1 genes in Fujian province[J]. Shi Jie Hua Ren Xiao Hua Za Zhi, 2009, 17(9):886-890

[20] M, O’brien SJ. The influence of HLA genotype on AIDS[J]. Annu Rev Med, 2003, 54:535-551

[21] MOHC, UNADS, WHO.China's HIV/AIDS epidemic estimates in 2011[J]. Chin J ADIS\&STD, 2011, 18(1):15

[22] NCAIDS/STD, CCDC.The National AIDS epidemic and major prevention and treatment progress in Nov[J]. 2014. Chin J ADIS\&STD, 2015, 21(02):87

[23] Odone A, Amadasi S, White RG, et al. The impact of antiretroviral therapy on mortality in HIV-1 positive People during tuberculosis treatment:a systematic review and meta-analysis[J].PLoS One, 2014, 9(11):112017

(Received 03 May 2018, Revised 28 May 2018, Accepted 02 June 2018) 